\title{
Optimization of Yttrium-90 Bremsstrahlung Imaging with Monte Carlo Simulations
}

\author{
Erwann Rault \\ Supervisor(s): Ignace Lemahieu, Stefaan Vandenberghe, Steven Staelens
}

\section{INTRODUCTION}

$\mathrm{T}$ ARGETED radionuclide therapy (TRT) is an emerging technique for cancer treatment. It uses the properties of some pharmaceuticals to fix in specific region of the body to deliver locally a lethal dose of radiation. Due to its physical properties, ${ }^{90} \mathrm{Y}$ is a good candidate for this therapy. Its high incidence of $\beta$ - emission optimizes the dose deposition within the targeted cells while its low incidence of photons emission minimizes the dose received by other organs. This low gamma emission limits however the imaging of the spatial distribution of the pharmaceutical in the body.

The bremsstrahlung interactions of the emitted $\beta$ - electrons in the body produces a continuous spectrum of photons that can be used for imaging [1]. Previous results show the importance of using a medium energy (MEGP) collimator together with a low energy window (50 to $150 \mathrm{keV}$ ) to minimize contamination and maximize the sensitivity while imaging bremsstrahlung photons. The aim of this study was, based on Monte Carlo simulations, to evaluate the efficiency of basic correction techniques, for contamination and detector blurring, in terms of contrast and noise.

\section{Materials AND Methods}

\section{A. Simulations}

The Philips AXIS system was simulated with the GATE [2] (Geant4 Application for Tomo-

E. Rault is with the Electronic and Information Systems Department, Ghent University (UGent), Gent, Belgium. E-mail: Erwann.Rault@UGent.be . graphic Emission) package. A previously developed fast bremsstrahlung photons generator [3] was used in this study to speed up the emission of the bremsstrahlung photons.

\section{B. Image reconstruction}

All images were reconstructed with the MLEM algorithm from 90 projections of the phantom. The images were reconstructed in $128 * 128 * 128$ matrices and the pixel size was set to $3.67 \mathrm{~mm}$.

Photons that scatter in the phantom or in the detector, as well as photons penetrating the collimator are going to induce false information in the sinogram and need to be corrected for. The background subtraction technique (Bgd. Sub.), used in PET and previously described by Karp et al. [4], was investigated in this study.

The distance dependent blurring of the detector (Det. Mod.) was also included in the reconstruction process. A system matrix was estimated by the simulation of multiple point sources at increasing distances from the collimator, and was then incorporated in both forward and back projections of the MLEM algorithm.

\section{Jaszczak phantom}

The Jaszczak phantom was used to evaluate the noise and the contrast recovery for the different reconstruction techniques. The simulated phantom was a cylinder homogeneously filled with activity and containing five hot lesions of respectively 19.1, 25.4, 31.8, 38 and $45.6 \mathrm{~mm}$ diameter and a cold lesion of $45.6 \mathrm{~mm}$ diameter (contrast: 8:1). 
The noise was defined as the normalized standard deviation of the pixels in the background region. It was computed on homogeneous slices of the phantom and from pixels situated at the same distance from the center of the phantom than the lesions to avoid the effect of attenuation.

The contrast recovery (CR) was computed using the following equation:

$$
C R=\frac{\mu_{\text {lesion }}-\mu_{b g d}}{\mu_{\text {bgd }}} * \frac{1}{C-1}
$$

where $\mu_{\text {lesion }}$ represents the mean value in the lesion and $m u_{b g d}$ the mean value in the background. $C$ is the theoretical contrast.

\section{RESUlts}

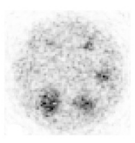

Normal

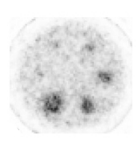

Bgd Sub.

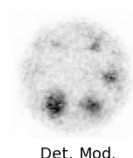

Det. Mod.

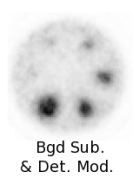

\& Det. Mod.
Figure 1. Reconstructed images.

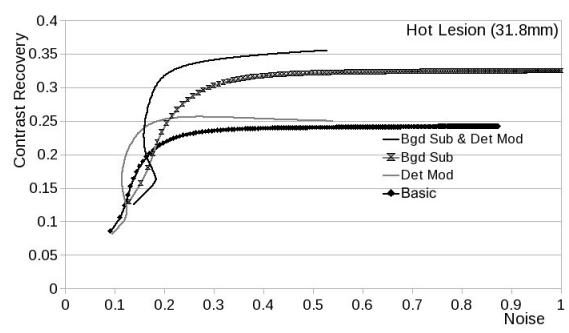

Figure 2. Contrast to Noise curves for the hotspot of $31.8 \mathrm{~mm}$.

Reconstructed images of the phantom are shown in Fig. 1. The contrast recovery curves show the same behavior for all lesion sizes (hot and cold lesions). The curves corresponding to the $31.8 \mathrm{~mm}$ hot lesion are shown in Fig. 2. For this lesion, the detector modeling (Det Mod) improves the contrast recovery of about $13 \%$ for $25 \%$ noise in the background. The background subtraction method (Bgd Sub) improves the contrast recovery of $30 \%$, while the use of both methods rises the contrast by $52 \%$. For the cold lesion, the use of both methods improves the contrast of $82 \%$.

\section{Discussion}

The background subtraction technique gives the best improvement in terms of contrast recovery. This method is however enhancing the noise in the images. This is the drawback of sinogram based scatter subtraction techniques. One possible solution could be to integrate the scatter estimation inside the forward projection of MLEM. This would ensure a better noise behavior during the reconstruction process.

All results presented in this study are based on a simple geometry of the phantom. Without any homogeneity, the contamination is well approximated with the background subtraction technique. For more complex geometries, the spatial distribution of the scatter would be more difficult to assess.

\section{CONCLUSiON}

This study presented an accurate method for the reconstruction of bremsstrahlung images. This method, based on a contamination background removal and on a resolution modeling of the camera in both forward and back projections, can improve the contrast of $52 \%$ for a hot lesion of $31.8 \mathrm{~mm}$ and of $82 \%$ for a cold lesion given the same amount of noise in the images.

\section{REFERENCES}

[1] S. Shen, G. L. DeNardo, A. Yuan, D. A. DeNardo, and S. J. DeNardo, "Planar gamma camera imaging and quantitation of yttrium-90 bremsstrahlung.," J Nucl Med, vol. 35, no. 8, pp. 1381-1389, Aug 1994.

[2] S. Jan, G. Santin, D. Strul, and S. Staelens et al., "GATE: a simulation toolkit for PET and SPECT.," Phys Med Biol, vol. 49, no. 19, pp. 4543-4561, Oct 2004.

[3] E. Rault, S. Vandenberghe, S. Staelens, J. De Beenhouwer, , and I. Lemahieu, "Fast simulation of yttrium-90 bremsstrahlung with gate," to be published in nimA, 2008.

[4] J. S. Karp, G. Muehllehner, D. A. MankofF, C. E. Ordonez, J. M. Ollinger, M. E. DaubeWitherspoon, A. T. Haigh, and D. J. Beerbohm, "Continuous-slice penn-pet: a positron tomograph with volume imaging capability.," $\mathrm{J} \mathrm{Nucl}$ Med, vol. 31, no. 5, pp. 617-627, May 1990. 